\title{
Letter to the editor. Group A streptococcal infections during the seasonal influenza outbreak 2010/11 in South East England
}

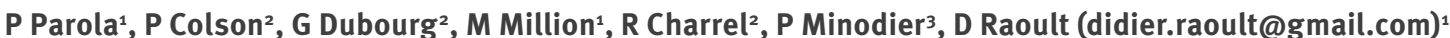

1. Infectious Diseases and Tropical Medicine Unit, University Hospitals of Marseille, Marseille, France

2. Fédération de Bactériologie-Hygiène-Virologie, Pôle des Maladies Infectieuses et Tropicales Clinique et Biologique, University Hospitals of Marseille, Timone Hospital, Marseille France

3. Urgences Pédiatriques, North University Hospital, Marseille, France

Citation style for this article:

Parola P, Colson P, Dubourg G, Million M, Charrel R, Minodier P, Raoult D. Letter to the editor. Group A streptococcal infections during the seasonal influenza

outbreak 2010/11 in South East England. Euro Surveill. 2011;16(11):pii=19816. Available online: http://www.eurosurveillance.org/ViewArticle.aspx?Articleld=19816

Article published on 17 March 2011

To the editor: We read with great interest the recent article about invasive Group A Streptococcus (GAS) infections associated with influenza B in England by Scaber et al. [1]. Indeed, since 2002 the Clinical Microbiology Laboratory of University Hospitals in Marseille, France, has implemented a tool for the weekly surveillance of microbiological data (called EPIMIC), which consists in a simple warning program using Microsoft Excel software. Both the numbers of samples received and of pathogens diagnosed are compared to historical data as soon as they are entered. Any significant increase beyond the critical threshold, defined by the mean of historical data plus two standard deviations (SDs), generates a signal allowing to detect abnormal and seasonal events in infectious diseases [2].

Recently, we have been alerted by an abnormal increase of invasive Group A Streptococcus (GAS) infections detected at the Point Of Care Laboratories of two main Marseille University hospitals (Timone and North hospitals), using rapid antigen detection (RAD) tests on throat swabs. In these two sites and during the three past years (2008-2010), the mean weekly number of GAS detection was six and four, respectively. Between 15 January, and 15 February, 143 RAD tests for GAS infections were positive in patients consulting at the emergency wards, including 98 at La Timone (69\%) and 44 at Hospital Nord (31\%). These patients had a mean age of 8.6 years (median, 5 years). At the beginning of February 2011, the number of positive GAS was higher than the critical threshold in both sites (mean +2 SDs), being about three times higher compared to the mean value. The number of samples to be tested also increased about the critical threshold.

When this alert was transmitted to the pediatricians working at the emergency wards of both hospitals, they reported to have examined an unusual number of children presenting with both influenza-like symptoms, in the context of seasonal influenza outbreak in France, and pharyngitis with GAS RAD positive testing.
At the same time, Scaber et al. reported their series of cases of invasive GAS co-infection with influenza B [1]. Therefore, we investigated retrospectively the association of GAS detection using the RAD test with influenza virus detection by the rapid influenza diagnostic test (RIDT) and real-time RT-PCR assays (rtRT-PCR) in naso-pharyngeal specimens [3]. From 1 January to 28 February, a total of 227 samples tested positive for GAS, and influenza tests were requested by clinicians in 74 of them. A total of 23 co-infections with influenza virus were identified (31\%), including 15 with influenza B virus, six with influenza A (not subtyped) and two with influenza $A\left(\mathrm{H}_{1} \mathrm{~N}_{1}\right)$ 2009. We also investigated the number of invasive GAS by checking the number of GAS positive blood cultures. From January 2007 through February 2011, 30 GAS positive blood cultures were identified in our laboratory, including 10 between 1 October, 2010 and 28 February, 2011 (p<0.05; Fisher and Yates tests, considering the number of blood culture samples received at the laboratories). As it can be considered that our laboratories cover a population of 600,000 persons living in Marseille and the surroundings, the incidence of invasive GAS in the last five months could be estimated at 1.6 per 100,000 population.

We provide here microbiological evidence of concurrent influenza viral infection in almost a third of children with GAS infections. It was a remarkable finding that over half of the 23 samples testing positive for influenza were influenza $B$. The high proportion of confirmed influenza $B$ in our series, even if in a small sample size, is striking, regarding the potential morbidity and mortality associated with influenza $B$ virus in the context of co-infection with invasive GAS, as recently reported [1].

Our warning and investigation resulted from the implementation of a surveillance tool to detect abnormal events in infectious disease. This method of surveillance may lead to other surprising discoveries. 


\section{Acknowledgements}

We acknowledge the kind help of $\mathrm{H}$ Richet in analysing the data.

\section{References}

1. Scaber I, Saeed S, Ihekweazu C, Efstratiou A, McCarthy N, O Moore E. Group A streptococcal infections during the seasonal influenza outbreak 2010/11 in South East England. Euro Surveill. 2011;16(5). pii:19780. Available from: http://www. eurosurveillance.org/ViewArticle.aspx?Articleld=19780

2. Anderson DJ, Richet H, Chen LF, Spelman DW, Hung YJ, Huang AT, et al. Seasonal variation in Klebsiella pneumoniae bloodstream infection on 4 continents. J Infect Dis. 2008;197(5):752-6.

3. Nougairede A, Ninove L, Zandotti C, de L, X, Gazin C, Drancourt $M$, et al. Point of care strategy for rapid diagnosis of novel A/ $\mathrm{H} 1 \mathrm{~N}_{1}$ influenza virus. PLoS One. 2010;5(2):e9215. 